\title{
Abstracts from Nippon Eiseigaku Zasshi (Japanese Journal of Hygiene) vol. 65 no. 3
}

(C) The Japanese Society for Hygiene 2010

\author{
Health Effects of Asian Dust Events: A Review \\ of the Literature
}

Nippon Eiseigaku Zasshi, 65, 413-421 (2010)

Masahiro Hashizume ${ }^{1}$, Kayo Ueda ${ }^{2}$, Yuji Nishiwaki ${ }^{3}$, Takehiro Michikawa ${ }^{3}$ and Daisuke Onozuka ${ }^{4}$

${ }^{1}$ Institute of Tropical Medicine, Nagasaki University

${ }^{2}$ National Institute for Environmental Studies

${ }^{3}$ Department of Preventive Medicine and Public Health, School of Medicine, Keio University

${ }^{4}$ Fukuoka Institute of Health and Environmental Sciences

Asian dust, called 'kosa' in Japan, is the long-range transport of atmospheric pollutants originating from the desert areas of China and Mongolia. Although Asian dust has a long history of appearing in Japan, it is only quite recently that there is increasing concern for its possible adverse health effects. We reviewed the epidemiologic evidence of potential health effects of Asian dust events. PubMed was used to search for the following keywords: Asian dust, yellow sand, desert dust, dust storm, sandstorm, mortality, death, morbidity, hospitalization, hospital admission, health, pulmonary and respiratory. The search was limited to the epidemiologic studies published between January 1980 and May 2009. JMEDPlus was used to search for Japanese literature. Seventeen studies were retrieved from PubMed and one study from JMEDPlus. In addition, one study was identified for reviewing from the references of another study. In total, we identified 19 epidemiologic studies ( 3 for mortality, 13 for hospital visits or admissions and 3 for respiratory functions or symptoms) mainly from Taiwan and Korea. There were many combinations of outcomes and lagged exposures examined, and some suggested possible associations of dust exposure with an increase in mortality and hospital visits and admissions due to cardiovascular and respiratory diseases, whereas the rest of the studies did not show statistically significant associations. The evidence from these studies was limited because exposure assessments were inadequately described and potential confounders were insufficiently controlled. Well-designed epidemiological studies are required to clarify any potential health effects of Asian dust events in Japan.

\section{Evaluation of Epidemiological Studies of Intestinal Bacteria that Affected Occurrence of Colorectal Cancer: Studies of Prevention of Colorectal Tumors by Dairy Products and Lactic Acid Bacteria}

Nippon Eiseigaku Zasshi, 65, 422-446 (2010)

Atsuko Kawano ${ }^{1}$, Hideki Ishikawa ${ }^{2}$,

Tomiyo Nakamura ${ }^{3}$ and Koichi Kono ${ }^{1}$

${ }^{1}$ Division of Preventive and Social Medicine, Department of Hygiene and Public Health, Osaka Medical College

${ }^{2}$ Department of Molecular-Targeting Cancer Prevention, Kyoto Prefectural University of Medicine

${ }^{3}$ Department of Molecular Pathology, Osaka University, Graduate School of Medicine and Health Science

Environmental factors have been consistently associated with colon cancer risk. In particular, consumption of Western-style diet including red meat is the most widely accepted etiologic risk factor. It has been reported that dietary factors change the proportion of intestinal flora, and it also affects the composition of fecal bile acids and the intestinal activity of some mutagens. In addition, it was suggested that modulating the composition of intestinal flora may reduce the occurrence of colorectal cancer. In this review, we present the clinical studies on the association between intestinal flora and the risk of colorectal cancer that have been carried out to date. The clinical studies of intestinal bacteria related to colorectal cancer risk have not shown consistent results so far, compared with the accomplishments of some basic studies. On the other hand, it was suggested in some clinical studies that lactic acid bacteria reduce the occurrence of colorectal cancer. 
Nationwide Study of Sick House Syndrome:

Comparison of Indoor Environment of Newly Built Dwellings between Sapporo City and Southern Areas Including Those in Honshu and Kyushu

Nippon Eiseigaku Zasshi, 65, 447-458 (2010)

Ayako Kanazawa $^{1}$, Yasuaki Saijo ${ }^{2}$, Masatoshi Tanaka ${ }^{3}$, Takesumi Yoshimura ${ }^{4}$, Hisao Chikara ${ }^{4}$,

Tomoko Takigawa ${ }^{5}$, Kanehisa Morimoto ${ }^{6}$,

Kunio Nakayama ${ }^{6}$, Eiji Shibata ${ }^{7}$ and Reiko Kishi ${ }^{1}$

${ }^{1}$ Department of Public Health Sciences,

Hokkaido University Graduate School of Medicine

${ }^{2}$ Department of Health Science, Asahikawa Medical College

${ }^{3}$ Fukushima College

${ }^{4}$ Fukuoka Institute of Health and Environmental Sciences

${ }^{5}$ Department of Public Health, Okayama University Graduate School

of Medicine, Dentistry and Pharmaceutical Sciences

${ }^{6}$ Department of Social and Environmental Medicine,

Osaka University Graduate School of Medicine

${ }^{7}$ Department of Health and Psychosocial Medicine,

Aichi Medical University School of Medicine

Objectives: The aims of this study are to evaluate the associations between residential factors and sick house syndrome (SHS) in a cold region and in a temperate region in Japan.

Methods: A questionnaire survey was conducted on dwellings aged within 7 years from 2003 to 2004 in Sapporo and 'Honshu and northern Kyushu' including Fukushima, Aichi, Osaka, Okayama, and Kitakyushu. The questionnaire consisted of questions about the characteristics of dwellings, manner of living, and health problems of occupants. SHS was identified from the 'yes' responses to the questions on the five items on nasal, throat and respiratory, eyes, skin, and general symptoms that appeared frequently or sometimes at home and improved upon leaving the home.

Results: The dwellings with the SHS problem, where at least one inhabitant reported any symptoms of SHS, were $4.7 \%$ in Sapporo City and $3.3 \%$ in Honshu and northern Kyushu. Regarding ventilation, the principal method was the enforced ventilation in Sapporo and ventilation from open windows in Honshu and northern Kyushu. The sums of five signs of dampness were $0.8 \pm 1.0$ in Sapporo and $1.3 \pm 1.0$ in Honshu and northern Kyushu. The elevated scores were accompanied by the increased percentage of SHS in both groups. Condensation on both window panes and walls was strongly associated with SHS.

Conclusions: Differences were found in the ventilation method and in the dampness state between Sapporo and Honshu and northern Kyushu. In both groups, the dampness state was associated with SHS.

\section{Evaluation of Vaccination Strategies to Suppress a Novel Influenza Pandemic Using an Individual-Based Model}

Nippon Eiseigaku Zasshi, 65, 459-466 (2010)

Tomoko Morimoto and Hirofumi Ishikawa

Department of Human Ecology, Graduate School of Environmental Science, Okayama University

Objectives: In this study, we aimed to evaluate vaccination strategies with regard to the impact of prioritization, coverage, and a delay in the vaccination program against a novel influenza pandemic with high-level fatality equivalent to Spanish flu using an individual-based model (IBM). Vaccination is one of the main measures to prevent infection, a serious condition, or death.

Methods: We constructed an IBM for the transmission of a novel influenza virus utilizing personal information on the basis of demographic data from Sapporo City, thereby resulting in a more realistic model. We designed simulations for scenarios along the guidelines of the Ministry of Health, Labour and Welfare of Japan, which requests the prioritization of vaccination of pregnant women and persons with underlying diseases, among whom influenza would cause higher fatality than among healthy persons, infants, or their parents, as well as school-aged and old-aged persons.

Results: A vaccination program fully taking into account the ordered priority groups would more effectively reduce the number of deaths in the priority groups and also the total number of deaths in comparison with a program shortening the transition time to the next priority or a non-priority group. A delay in the vaccination program would lower the effectiveness of reducing the numbers of patients and deaths.

Conclusions: According to the simulation-based results, when vaccination programs scheduled on the basis of priority groups start 90 and 150 days after outbreak, the total numbers of patients would be reduced to one-third-one-half, and two-thirds, respectively, in comparison with baseline of no vaccination, which leads to the necessity to conduct a vaccination program as soon as possible.

\section{Study of Experiment on Leaching of Bisphenol A from Infant Books to Artificial Saliva}

Nippon Eiseigaku Zasshi, 65, 467-470 (2010)

Junko Sajiki, Ryoko Yanagibori and Yaeko Kobayashi

Chiba Prefectural Institute of Public Health

Objective: To assess the risk of bisphenol A (BPA) exposure when infants suck or chew infant books, the concentration of BPA leaching from infant books published by Japanese makers to artificial saliva was measured.

Methods: The concentration of BPA leaching from 10 infant books to $15 \mathrm{ml}$ artificial saliva or water was measured at $37^{\circ} \mathrm{C}$ for $20 \mathrm{~h}$. BPA concentration was measured by high-performance liquid chromatography-electrochemical detection (HPLC-ECD) with solid-phase extraction.

Results: BPA was leached from all books when pieces of them were dipped both into saliva and water for $20 \mathrm{~h}$. The highest concentration of BPA leaching from one out of 10 books was $43.4 \mathrm{ng} / \mathrm{ml}$ (for $2 \mathrm{~h}$ ) in saliva, which was estimated to be approximately $0.052 \mu \mathrm{g} / \mathrm{kg}$ body weight/day for infants aged 6-10 months.

Conclusion: As BPA has endocrine-disrupting effects and poses higher risks in infants than in adults, it is desired to reduce BPA use in the printing of infant books from the viewpoint of child health. 